\title{
Comportamiento de índices no lineales de variabilidad del ritmo cardiaco al inhibir el sistema nervioso autónomo
}

\author{
Juan Bolea ${ }^{1,2}$, Esther Pueyo ${ }^{2,1}$, Pablo Laguna ${ }^{1,2}$, Raquel Bailón ${ }^{1,2}$ \\ ${ }^{1}$ Grupo de Tecnologías de la Comunicación (GTC) \\ Instituto de Investigación en Ingeniería de Aragón (I3A). \\ Universidad de Zaragoza, Mariano Esquillor s/n, 50018, Zaragoza, Spain. \\ Tel. +34-976762707, Fax +34-976762043, e-mail: jbolea@unizar.es \\ ${ }^{2}$ Centro de Investigación Biomédica en Red - Bioingeniería, Biomateriales y Nanomedicina (CIBER-BBN)
}

\begin{abstract}
Indices no lineales, basados en la teoría del caos, vienen siendo aplicados al estudio de la variabilidad del ritmo cardiaco en las últimas décadas como alternativa a los índices lineales tradicionalmente empleados para la cuantificación de información clínica relevante. El presente estudio analiza la interpretación fisiológica de varios de estos índices no lineales a partir de registros electrocardiográficos en los que se bloquea alternativamente una de las ramas del sistema nervioso autónomo (simpática o parasimpática) mediante el uso de fármacos.
\end{abstract}

\section{Introducción}

El estudio de la variabilidad del ritmo cardiaco (HRV, del inglés Heart Rate Variability) es una técnica no invasiva que permite evaluar la regulación de la actividad cardiaca por el sistema nervioso autónomo. En [1] se estandariza el uso e interpretación fisiológica de algunas técnicas de análisis de la HRV. Entre ellos se encuentran los métodos lineales, principalmente características temporales (como la media y la desviación estándar), y frecuenciales. Para el análisis espectral se definen dos bandas frecuenciales, LF (Low Frequency) $(0.04-0.15 \mathrm{~Hz})$ relacionada con las modulaciones simpática y parasimpática y HF (High Frequency $\quad(0.15-0.4 \mathrm{~Hz}) \quad$ relacionada fundamentalmente con la actividad parasimpática.

En las últimas décadas el uso de índices no lineales basados en la teoría del caos, como approximate entropy (ApEn) [2] y correlation dimension $\left(D_{2}\right)$ [3], está siendo cada vez más extendido. Estudios previos [4] han demostrado que la combinación de estos índices con los clásicos proporciona un incremento en la capacidad estratificadora del análisis de HRV en el contexto de distintas patologías. Sin embargo, la relación de los índices no lineales y los mecanismos fisiologícos subyacentes no está bien establecida.
En este trabajo, se estudian los índices no lineales $A p E n$ y $D_{2}$ en sujetos a los que se les inhibe selectivamente una de las ramas del sistema nervioso autónomo. Los índices frecuenciales servirán como referencia para evaluar los cambios provocados por los bloqueos en los índices no lineales basados en caos. Asimismo, se proporcionará una interpretación fisiológica de dichos cambios.

\section{Base de datos}

La base de datos seleccionada está formada por 13 sujetos (19-38 años) sanos, sin histórico previo de enfermedad cardiopulmonar, a los que se les inhibió parcialmente el sistema nervioso autónomo mediante dos fármacos. A 7 de ellos se les administró atropina $(0.03 \mathrm{mg} / \mathrm{kg})$ como bloqueador del sistema parasimpático (G-A) mientras que al resto se les inhibió el sistema simpático con una dosis de propranolol (0.2 mg/kg) (G-P).

Se les registró el electrocardiograma (ECG) durante 7 minutos (con una frecuencia de muestreo de 360 $\mathrm{Hz}$ ), primero en condiciones de control y posteriormente trás 10 minutos de haber sido administrado el fármaco. Ambos registros se realizaron con el sujeto en posición supina. Una descripción más amplia acerca de esta base de datos puede encontrarse en [5].

\section{Series y análisis de HRV}

Una baja frecuencia de muestreo en la señal ECG puede producir que la determinación de la posición de los latidos esté desplazada unas pocas muestras, añadiendo variabilidad espuria no deseada. Para reducir este problema se interpolaron las señales ECG a $1080 \mathrm{~Hz}$, antes de la aplicación del detector de QRS. Los latidos se detectaron mediante una técnica basada en la transformada wavelet, obteniéndose las posiciones de los latidos, $t_{i}$. Con éstas se computó el tacograma cardiaco, que se define como la serie de intervalos de tiempo entre 
un latido, $t_{i}$ y el anterior, $t_{i-1}$. Utilizando el modelo IPFM (del inglés, integrated pulse frequency modulation) sobre el tacograma se estimó la señal moduladora del ritmo, que se considera que representa la acción del sistema nervioso autónomo, y por tanto es de utilidad para el estudio de la HRV. Esta señal moduladora se interpoló a $4 \mathrm{~Hz}$ y se utilizó para extraer las características frecuenciales de la HRV.

Por otro lado, los índices no lineales, $A p E n$ y $D_{2}$, usan la serie de $R R$ para caracterizar al sistema que genera la serie en términos de regularidad y de complejidad, respectivamente. ApEn fue calculada siguiendo el trabajo de [2], con $m=2$ y $r=$ $0.15 S T D$, siendo $m$ la dimensión de los vectores reconstruidos, $r$ la tolerancia y STD la desviación estándar. Para el cómputo de $D_{2}$, nos basamos en [3], que introduce el uso de sigmoides para ajustar las curvas de sumas de correlación. En este caso $m_{\max }=16$ y $r \in[0.013]$ STD en pasos de 0.01STD.

El análisis de HRV se realiza en los 5 últimos minutos de cada registro de ECG. El test estadístico de Wilcoxon se aplica para ver cambios significativos comparando los índices en control y con el bloqueo autonómico correspondiente.

Los resultados obtenidos mediante el análisis frecuencial y los métodos no lineales se muestran en la Tabla 1.

\section{Discusión y conclusiones}

La administración de propranolol produce una inhibición del sistema simpático. Las potencias en LF y HF no experimentan cambios pese al bloqueo. Por otro lado en los índices no lineales se observan tendencias de cambio. Sin embargo estos cambios no tienen significancia estadística. Estos resultados pueden deberse a que la actividad simpática en posición supina está inhibida siendo inapreciable el efecto del fármaco.
Cuando el sistema parasimpático es el inhibido por la administración de la atropina, los cambios en todos los índices son significativos hacia una reducción de su valor. La casi ausencia de la alta frecuencia en la señal de variabilidad se traduce en una variabilidad más regular y menos compleja.

Pese al reducido número de sujetos considerados en este trabajo, lo que supone una limitación en los análisis estadísticos, se observan tendencias de cambio en los índices no lineales por efecto de los cambios en el sistema nervioso autónomo. Un estudio más extenso sobre esta misma base de datos, considerando otras posiciones de los sujetos así como distintas combinaciones en la administración de fármacos, contribuirá a proporcionar una mejor interpretación de los índices no lineales estudiados.

\section{REFERENCIAS}

[1]. TASK FORCE OF ESC and NASPE. Heart rate variability, standards of measurement, physiological interpretation, and clinical use. Circulation. 1996, 93(5), 1043-1065.

[2]. PINCUS, S. and SINGER, B.H. Randomness and degrees of irregularity. Proceedings of the National Academy of Sciences. 1996, 93(5), 2083-2088.

[3]. BOLEA, J. et al. Methodological framework for estimating the correlation dimensión in HRV signals. Computational and Mathematical Methods in Medicine. 2014, 129248.

[4]. ALETTI, F. et al. Heart rate variability in children with cyanotic and acyanotic congenital heart disease: Analysis by spectral and non-linear índices. In: Engineering in Medicine and Biology Society (EMBC), Annual International Conference of the IEEE. New York: IEEE, 2012, pp. 4189-4192.

[5]. SAUL, J.P. et al. Transfer function analysis of the circulation: unique insights into cardiovascular regulation. American Journal of Physiology-Heart and Circulatory Physiology. 1991, 261(4), H1231H1245.

Tabla 1. Resultados de los índices frecuenciales y no lineales evaluados en el estudio de HRV. Los valores están representados por mediana \pm rango intercuartílico.* $\mathbf{p}<0.05$ mediante el test estadístico de Wilcoxon respecto de la condición de control.

\begin{tabular}{|c|c|c|c|c|}
\multirow{2}{*}{ por mediana \pm rango intercuartílico. $\mathbf{p}<\mathbf{0 . 0 5}$ mediante el test estadistico de Wilcoxon respecto de la condicion de control. } \\
\cline { 2 - 5 } & \multicolumn{2}{|c|}{ G-P $(\mathrm{N}=6)$} & Control & Atropina \\
\cline { 2 - 5 } & Control & Propranolol & $434 \pm 629$ & $21 \pm 18 *$ \\
\hline PLF [adim] $\left(\cdot 10^{-5}\right)$ & $186 \pm 321$ & $238 \pm 314$ & $178 \pm 207$ & $1.52 \pm 2.98 *$ \\
\hline PHF [adim] $\left(\cdot 10^{-5}\right)$ & $129 \pm 47$ & $136 \pm 92$ & $0.70 \pm 0.18$ & $0.21 \pm 0.20 *$ \\
\hline$A p E n(2,0.15)$ & $0.85 \pm 0.49$ & $0.72 \pm 0.20$ & $5.22 \pm 1.25$ & $3.38 \pm 0.63 *$ \\
\hline$D_{2}$ & $5.44 \pm 0.49$ & $6.41 \pm 1.09$ & \\
\hline
\end{tabular}

